\title{
Classification of the Ricci Tensor*
}

\author{
Garry Ludwig and Gerry Scanlan \\ Department of Mathematics, University of Alberta, Edmonton, Alberta, Canada
}

Received November 10, 1970

\begin{abstract}
This paper contains a classification of the Ricci tensor $R_{\alpha \beta}$. The method of derivation is analogous to the spinor version of the Petrov classification of the Weyl tensor. It is shown how the various classes are related to the number and type of eigenvectors and eigenvalues of $R_{\alpha \beta}$. The classification is useful in the geometrization of various fields. The case of a real scalar field is treated in detail.
\end{abstract}

\section{Introduction}

In a recent paper [1] the "already unified" field theory of Rainich, Misner, and Wheeler, including the null case, was derived using a classification of the Ricci tensor which is somewhat analogous to the spinor version of the Petrov classification $[2,3]$ of the Weyl tensor. However, the classification was given only as far as necessary for the remainder of the paper. Here we shall develop it in detail. As a further application we shall consider the geometrization of a massless real scalar field. Results obtained previously by Brill [4] and Penney [5] are found to be incomplete.

In Section 2 we shall explain the notation. Section 3 deals with the classification of the Ricci tensor $R_{\alpha \beta}$ and Section 4 with the relation of this classification to the number and type of eigenvectors and eigenvalues of $R_{\alpha \beta}$ [6]. In Section 5 we apply this classification to the problem of geometrization of a massless real scalar field. In the appendix we exhibit canonical forms for the dyad components of the tracefree Ricci tensor for various classes.

\section{Notation}

The notation used is the same as in Ref. [1]. Tensor indices are given by small Greek letters and run from 1 to 4 . Spinor indices are denoted by capital Latin letters and run from 1 to 2 . The summation convention is used throughout. Ordinary differentiation is indicated by a comma,

$\star$ Supported in part by the National Research Council of Canada. 
covariant differentiation by a semi-colon. Round brackets around suffices denote symmetrization, square brackets denote anti-symmetrization. A bar over a quantity denotes the complex conjugate of that quantity. Complex conjugation of a spinor is indicated by a bar over the letter and a dot over the spinor index. The correspondence between a tensor and its spinor equivalent is denoted by $\leftrightarrow$. The signature of space-time is taken to be -2 . The Ricci tensor $R_{\mu \gamma}$ and the Ricci scalar $R$ are defined as in Ref. [7]. Finally, we write $R \cdot R$ instead of $R_{\alpha} R^{\alpha}$.

\section{Classification of $\boldsymbol{R}_{\alpha \beta}$}

To classify the Ricci tensor $R_{\alpha \beta}$ we consider the expression $R_{\alpha \beta} l^{\alpha} l^{\beta}$ for arbitrary null vectors $l^{\alpha}$. Since this expression equals $S_{\alpha \beta} l^{\alpha} l^{\beta}$, where $S_{\alpha \beta}$ is the tracefree part of $R_{\alpha \beta}$, two Ricci tensors which differ by a multiple of the metric tensor $g_{\alpha \beta}$ will belong to the same class.

The spinor equivalent of $-\frac{1}{2} S_{\alpha \beta}$ is $\Phi_{A B \dot{W} \dot{X}}$. Its components are, as usual [7], denoted by $\Phi_{00}, \Phi_{11}, \Phi_{22}, \Phi_{01}, \Phi_{10}, \Phi_{02}, \Phi_{20}, \Phi_{12}, \Phi_{21}$, where the first three are real and $\Phi_{10}=\bar{\Phi}_{01}, \Phi_{20}=\bar{\Phi}_{02}, \Phi_{21}=\bar{\Phi}_{12}$. A real null vector $l^{\alpha}$ has associated with it a spinor $l^{A}$ defined by

Therefore,

$$
l_{\leftrightarrow}^{\alpha} \leftrightarrow \pm l^{A} \bar{l}^{\dot{x}} .
$$

$$
R_{\alpha \beta} l^{\alpha} l^{\beta}=-2 \Phi_{A B \dot{W} \dot{X}} l^{A} l^{B} \dot{l}^{\dot{W}} \dot{l}^{\dot{X}}=G l^{2} l^{2} \dot{l}^{2} \bar{l}^{2},
$$

where

and

$$
\begin{aligned}
G= & -2\left[\Phi_{00} c^{2} \bar{c}^{2}+2 \Phi_{01} c^{2} \bar{c}+2 \Phi_{10} c \bar{c}^{2}+\Phi_{02} c^{2}+\Phi_{20} \bar{c}^{2}\right. \\
& \left.+4 \Phi_{11} c \bar{c}+2 \Phi_{12} c+2 \Phi_{21} \bar{c}+\Phi_{22}\right],
\end{aligned}
$$

$$
c=\frac{l^{1}}{l^{2}} .
$$

The quartic $G$ defined by Eq. (3.1) can always be written in the form

$$
2 G=G_{1} G_{2}+\bar{G}_{1} \bar{G}_{2},
$$

where $G_{1}$ and $G_{2}$ are two quadratic forms which are not necessarily Hermitian. For example,

$$
G_{1}=R_{1 \dot{1}} c \bar{c}+R_{1 \dot{2}} c+R_{2 \dot{1}} \bar{c}+R_{2 \dot{2}},
$$

where, in general, all of the coefficients are complex and independent and may be regarded as the dyad components of a complex vector $R_{\alpha}$. In a similar fashion $G_{2}$ determines a complex vector $S_{\alpha}$. The decomposition theorem Eq. (3.2) is equivalent to the statement that for each tracefree symmetric tensor $S_{\alpha \beta}$ there exist two complex vectors $R_{\alpha}$ and $S_{\alpha}$ (which 
are, however, not unique) such that

$$
2 S_{\alpha \beta}=R_{(\alpha} S_{\beta)}+\bar{R}_{(\alpha} \bar{S}_{\beta)}-\frac{1}{4} g_{\alpha \beta}(R \cdot S+\bar{R} \cdot \bar{S}) .
$$

The proof of this theorem is straightforward. If we compare coefficients on both sides of Eq. (3.2) we obtain the equations

$$
\begin{aligned}
& -4 \Phi_{00}=R_{1 \mathrm{i}} S_{1 \mathrm{i}}+\overline{R_{1 \mathrm{i}}} \overline{S_{1 \mathrm{i}}} \text {, } \\
& -8 \Phi_{01}=R_{1 \mathrm{i}} S_{1 \dot{2}}+R_{1 \dot{2}} S_{1 \mathrm{i}}+\overline{R_{1 \mathrm{i}}} \overline{S_{2 \mathrm{i}}}+\overline{R_{2 \mathrm{i}}} \overline{S_{1 \mathrm{i}}} \text {, } \\
& -4 \Phi_{02}=R_{1 \dot{2}} S_{1 \dot{2}}+\overline{R_{2 i}} \overline{S_{2 i}} \text {, } \\
& -16 \Phi_{11}=R_{1 \mathrm{i}} S_{2 \dot{2}}+R_{2 \dot{2}} S_{1 \mathrm{i}}+R_{1 \dot{2}} S_{2 \mathrm{i}}+R_{2 \dot{1}} S_{1 \dot{2}} \\
& +\overline{R_{1 \mathrm{i}}} \overline{S_{2 \dot{2}}}+\overline{R_{2} \dot{2}} \overline{S_{1 \mathrm{i}}}+\overline{R_{1 \dot{2}}} \overline{S_{2 \mathrm{i}}}+\overline{R_{2 \mathrm{i}}} \overline{S_{1 \dot{2}}} \text {, } \\
& -8 \Phi_{12}=R_{1 \dot{2}} S_{2 \dot{2}}+R_{2 \dot{2}} S_{1 \dot{2}}+\overline{R_{2 \dot{1}}} \overline{S_{2 \dot{2}}}+\overline{R_{2 \dot{2}}} \overline{S_{2 \dot{1}}} \text {, } \\
& -4 \Phi_{22}=R_{2 \dot{2}} S_{2 \dot{2}}+\overline{R_{2 \dot{2}}} \overline{S_{2 \dot{2}}} \text {. }
\end{aligned}
$$

It is easy to see that a solution always exists but will not be unique.

This decomposition theorem allows us to classify $R_{\alpha \beta}$ as follows. $R_{\alpha \beta}$ is in class $A$ if the decomposition may be taken to be

$$
G= \pm G_{1}^{2}
$$

for some Hermitian quadratic form $G_{1}$, corresponding to a real vector $R_{\alpha} . R_{\alpha \beta}$ is in class $B$ if the decomposition may be taken to be

$$
G=G_{1} G_{2}
$$

for some pair of Hermitian quadratic forms $G_{1}$ and $G_{2}$, corresponding to a pair of real and non-parallel vectors $R_{\alpha}$ and $S_{\alpha} . R_{\alpha \beta}$ is in class $C$ if the decomposition may be taken to be

$$
G= \pm G_{1} \bar{G}_{1}
$$

where $G_{1}$ is a non-Hermitian quadratic form corresponding to a complex vector $R_{\alpha}(R \cdot R \neq 0)$ which cannot be made real by multiplying by a phase factor. Class $D$ contains the remaining Ricci tensors.

That these four classes are disjoint follows from the fact that when $G$ factors as in $A, B$ or $C$ the factorization is essentially unique. The vector $R_{\alpha}$ in class $A$ is determined by $S_{\alpha \beta}$ uniquely except for sign. The vectors $R_{\alpha}$ and $S_{\alpha}$ in class $B$ are determined by $S_{\alpha \beta}$ up to the duality transformation

$$
\begin{aligned}
& R_{\alpha}^{\prime}=\kappa R_{\alpha}, \\
& S_{\alpha}^{\prime}=\kappa^{-1} S_{\alpha} \quad(\text { for } \kappa \text { real })
\end{aligned}
$$


also, $R_{\alpha}$ and $S_{\alpha}$ may be interchanged. In class $C$ the complex vector $R_{\alpha}$ is determined up to the duality rotation

$$
R_{\alpha}^{\prime}=e^{i \theta} R_{\alpha} \quad \text { (for } \theta \text { real); }
$$

also, $R_{\alpha}$ and $\bar{R}_{\alpha}$ may be interchanged.

The classification may be refined. Let us deal with class $A$ first. The sign appearing in Eq. (3.4) already divides $A$ into two subclasses, $A_{+}$and $A_{-}$. Since the invariant $R \cdot R$ is uniquely specified by $S_{\alpha \beta}$ we can classify the Ricci tensor further according as $R_{\alpha}$ is space-like $\left(A_{1}\right)$, time-like $\left(A_{2}\right)$ or null $\left(A_{3}\right)$.

If $R_{\alpha \beta}$ belongs to class $B$ it determines the signs of the invariants $R \cdot R$ and $S \cdot S$ (up to an interchange) as well as the invariant $R \cdot S$. The subclasses are obtained by considering the various possibilities for the signs of all three invariants. Another important invariant is $I$, defined by

$$
I=(R \cdot S)^{2}-(R \cdot R)(S \cdot S) .
$$

It is positive when the $R-S$ plane is time-like, negative when it is spacelike and zero when it is null.

In class $C, R_{\alpha \beta}$ determines a complex vector $R_{\alpha}$ up to a duality rotation Eq. (3.6) and an interchange of $R_{\alpha}$ and $\overline{R_{\alpha}}$. The vectors $S_{\alpha}$ and $T_{\alpha}$. defined by

$$
R_{\alpha}=S_{\alpha}+i T_{\alpha},
$$

may be chosen orthogonal since $R \cdot R$, given by

$$
R \cdot R=S \cdot S-T \cdot T+2 i S \cdot T,
$$

may be made real by a duality rotation. The invariant $I$, defined by

$$
I=\frac{1}{4}|R \cdot R|^{2}-\frac{1}{4}(R \cdot \bar{R})^{2}=(S \cdot T)^{2}-(S \cdot S)(T \cdot T),
$$

distinguishes between three subclasses. It is positive $\left(C_{1}\right)$ when the $S-T$ plane is time-like, zero $\left(C_{2}\right)$ when it is null and negative $\left(C_{3}\right)$ when it is space-like. Due to the choice of sign in Eq. (3.5) there are actually six subclasses for class $C$.

Tables 1 and 2 give a summary of the classification.

Table 1

\begin{tabular}{ll}
\hline Class & Decomposition \\
\hline$A_{ \pm}$ & $S_{\alpha \beta}= \pm\left[R_{\alpha} R_{\beta}-\frac{1}{4} g_{\alpha \beta} R \cdot R\right] ; R_{\alpha}$ is real. \\
$B$ & $S_{\alpha \beta}=R_{(\alpha} S_{\beta)}-\frac{1}{4} g_{\alpha \beta}(R \cdot S) ;$ \\
& $R_{\alpha}$ and $S_{\alpha}$ are real and not parallel. \\
$C_{ \pm}$ & $S_{\alpha \beta}= \pm\left[R_{(\alpha} \bar{R}_{\beta)}-\frac{1}{4} g_{\alpha \beta}(R \cdot \bar{R})\right] ; R \cdot R \neq 0$, \\
& $R_{\alpha}$ is a complex but not proportional to a real vector. \\
& $2 S_{\alpha \beta}=R_{(\alpha} S_{\beta)}+\bar{R}_{(\alpha} \bar{S}_{\beta)}-\frac{1}{4} g_{\alpha \beta}(R \cdot S+\bar{R} \cdot \bar{S}) ;$ \\
& $S_{\alpha \beta}$ cannot be written as in $A, B$ or $C$. \\
\hline
\end{tabular}


Table 2

\begin{tabular}{lllll}
\hline Class & \multicolumn{5}{l}{ Sign of the relevant invariants } \\
\cline { 2 - 5 } & $R \cdot R$ & $S \cdot S$ & $R \cdot S$ & $I$ \\
\hline$A_{1 \pm}$ & - & & & \\
$A_{2 \pm}$ & + & & & \\
$A_{3 \pm}$ & 0 & & & \\
$B_{1 a}$ & 0 & 0 & + & + \\
$B_{1 b}$ & 0 & 0 & - & + \\
$B_{2 a}$ & - & 0 & 0 & 0 \\
$B_{2 b}$ & - & 0 & + & + \\
$B_{2 c}$ & - & 0 & - & + \\
$B_{3 a}$ & - & - & 0 & - \\
$B_{3 b}$ & - & - & + & - \\
$B_{3 c}$ & - & - & - & - \\
$B_{3 d}$ & - & - & + & 0 \\
$B_{3 e}$ & - & - & - & 0 \\
$B_{3 f}$ & - & - & + & + \\
$B_{3 g}$ & - & - & - & + \\
$B_{4 a}$ & 0 & + & + & + \\
$B_{4 b}$ & 0 & + & - & + \\
$B_{5 a}$ & + & + & + & + \\
$B_{5 b}$ & + & + & - & + \\
$B_{6 a}$ & - & + & 0 & + \\
$B_{6 b}$ & - & + & + & + \\
$B_{6 c}$ & - & + & - & + \\
$C_{1 \pm}$ & & & & + \\
$C_{2 \pm}$ & & & & 0 \\
$C_{3 \pm}$ & & & & - \\
\hline
\end{tabular}

\section{Eigenvectors and Eigenvalues of $\boldsymbol{R}_{\alpha \beta}$}

The relation between the present classification and that in terms of the eigenvalues and eigenvectors of $R_{\alpha \beta}$ is shown in Table 3 .

The eigenvalues in the fourth column are numbered in the order in which the corresponding eigenvectors are listed in the third column. They are all real except where indicated. Eigenvectors corresponding to distinct eigenvalues are orthogonal. A space-like eigenvector is denoted by $s-l$, a time-like one by $t-l$. A $s-l(t-l$, null) plane of eigenvectors with the same eigenvalue is called an $s-l(t-l$, null) plane. The hyperplanes in class $A$ are also hyperplanes of eigenvectors. If $k_{\alpha}$ is a null eigenvector, $c$ is defined to be $R_{\alpha \beta} m^{\alpha} m^{\beta}$ where the null vector $m^{\alpha}$ is orthogonal to all other eigenvectors and satisfies $k_{\alpha} m^{\alpha}=1$. The quantity $b$ appearing in the last case is defined by $-R_{\alpha \beta} m^{\alpha} y^{\beta}$ where $y^{\alpha}$ is orthogonal to the space-like eigenvector and such that $y \cdot y=-1, y \cdot k=y \cdot m=0$. 
Table 3

\begin{tabular}{|c|c|c|c|}
\hline Class & $\begin{array}{l}\text { Segré } \\
\text { characteristic }\end{array}$ & Eigenvectors & Eigenvalues \\
\hline $\begin{array}{l}A_{1 \pm} \\
A_{2 \pm} \\
A_{3 \pm}\end{array}$ & $\begin{array}{l}{[1(111)]} \\
{[1(111)]} \\
{[(211)]}\end{array}$ & $\begin{array}{l}s-l \text {, hyperplane } \\
t-l \text {, hyperplane } \\
\text { null hyperplane }\end{array}$ & $\begin{array}{l}\lambda_{1} \lessgtr \lambda_{2}=\lambda_{3}=\lambda_{4} \\
\lambda_{1} \gtrless \lambda_{2}=\lambda_{3}=\lambda_{4} \\
\lambda_{1}=\lambda_{2}=\lambda_{3}=\lambda_{4} ; c \gtrless 0\end{array}$ \\
\hline $\begin{array}{l}B_{1 a, b} \\
B_{2 a} \\
B_{2 b, c} \\
B_{3 a, b, c} \\
B_{3 d, e} \\
B_{3 f, g} \\
B_{4 a, b} \\
B_{5 a, b} \\
B_{6 a, b, c}\end{array}$ & $\begin{array}{l}{[(11)(11)]} \\
{[(31)]} \\
{[2(11)]} \\
{[(11) 11]} \\
{[(21) 1]} \\
{[(11) 11]} \\
{[2(11)]} \\
{[(11) 11]} \\
{[(11) 11]}\end{array}$ & $\begin{array}{l}s-l \text { plane, } t-l \text { plane } \\
\text { null plane } \\
s-l \text { plane, null } \\
t-l \text { plane, } 2 s-l \\
\text { null plane, } s-l \\
s-l \text { plane, } s-l, t-l \\
s-l \text { plane, null } \\
s-l \text { plane, } s-l, t-l \\
s-l \text { plane, invariant } t-l \text { plane }\end{array}$ & $\begin{array}{l}\lambda_{1}=\lambda_{2} \lessgtr \lambda_{3}=\lambda_{4} \\
\lambda_{1}=\lambda_{2}=\lambda_{3}=\lambda_{4} \\
\lambda_{1}=\lambda_{2} \lessgtr \lambda_{3}=\lambda_{4} ; c \lessgtr 0 \\
\lambda_{3}<\lambda_{1}=\lambda_{2}<\lambda_{4} ; \lambda_{3}+i_{4} \risingdotseq 2 \lambda_{1} \\
\lambda_{1}=\lambda_{2}=\lambda_{3} \lessgtr \lambda_{4} ; c \gtrless 0 \\
\lambda_{1}=\lambda_{2} \lessgtr \lambda_{4} \lessgtr \lambda_{3} \\
\lambda_{1}=\lambda_{2} \lessgtr \lambda_{3}=\lambda_{4} ; c \gtrless 0 \\
\lambda_{1}=\lambda_{2} \lessgtr \lambda_{3} \lessgtr \lambda_{4} \\
\lambda_{1}=\lambda_{2} ; \lambda_{3}, \hat{\lambda}_{3} ; c \risingdotseq 0\end{array}$ \\
\hline $\begin{array}{l}C_{1 \pm} \\
C_{2 \pm} \\
C_{3 \pm}\end{array}$ & $\begin{array}{l}{[(11) 11]} \\
{[(21) 1]} \\
{[(11) 11]}\end{array}$ & $\begin{array}{l}s-l \text { plane, } s-l, t-l \\
\text { null plane, } s-l \\
t-l \text { plane, } 2 s-l\end{array}$ & $\begin{array}{l}\lambda_{3} \lessgtr \lambda_{1}=\lambda_{2} \lessgtr \lambda_{4} \\
\lambda_{4} \lessgtr \lambda_{1}=\lambda_{2}=\lambda_{3} ; c \gtrless 0 \\
\lambda_{3}, \lambda_{4} \lessgtr \lambda_{1}=\lambda_{2}\end{array}$ \\
\hline $\begin{array}{l}D_{1} \\
D_{2} \\
D_{3} \\
D_{4}\end{array}$ & $\begin{array}{l}{[1111]} \\
{[1111]} \\
{[211]} \\
{[31]}\end{array}$ & $\begin{array}{l}3 s-l, t-l \\
2 s-l, \text { invariant } t-l \text { plane } \\
\text { null, } 2 s-1 \\
s-l, \text { null }\end{array}$ & $\begin{array}{l}\lambda_{1} \lambda_{2}, \lambda_{3}, \lambda_{4} ; \text { all different } \\
\lambda_{1} \neq \lambda_{2}, \lambda_{3}, \hat{\lambda}_{3} \\
\lambda_{4} \neq \lambda_{1}=\lambda_{2} \neq \lambda_{3} \neq \lambda_{4} ; c \neq 0 \\
\lambda_{1} \neq \lambda_{2}=\lambda_{3}=\lambda_{4} ; b \neq 0\end{array}$ \\
\hline
\end{tabular}

The classification is complete. Types such as three space-like eigenvectors corresponding to three distinct eigenvalues cannot occur, as can easily be shown. For any possible type we can readily find the corresponding class and vice versa.

For example, if $R_{\alpha \beta}$ has two distinct pairs of real eigenvalues corresponding to a space-like plane and a time-like plane of eigenvectors respectively, then taking these planes to be the $x-y$ and $z-t$ planes we get

$$
R_{\alpha \beta}=-\lambda_{1} x_{\alpha} x_{\beta}-\lambda_{2} y_{\alpha} y_{\beta}-\lambda_{3} z_{\alpha} z_{\beta}+\lambda_{4} t_{\alpha} t_{\beta}
$$

with $\lambda_{1}=\lambda_{2} \neq \lambda_{3}=\lambda_{4}$ for eigenvalues. We can always add a multiple of $g_{\alpha \beta}$ to $R_{\alpha \beta}$ without changing the class to which $R_{\alpha \beta}$ belongs. This will leave the eigenvectors unchanged and increase all eigenvalues by that multiple. Thus, in our present example we may assume that $\lambda_{1}=\lambda_{2}=0$. We then have

where

$$
R_{\alpha \beta}=\lambda_{3}\left(z_{(\alpha}+t_{(\alpha)}\right)\left(t_{\beta)}-z_{\beta)}\right)=R_{(\alpha} S_{\beta)}
$$

$$
R \cdot R=S \cdot S=0, \quad R \cdot S=2 \lambda_{3} .
$$

Therefore, $R_{\alpha \beta}$ is in class $B_{1 a}$ if $\lambda_{3}>0$ and in class $B_{1 b}$ if $\lambda_{3}<0$. 
Conversely, if $R_{\alpha \beta}$ belongs to class $B_{1 a, b}$ then, apart from a multiple of $g_{\alpha \beta}, R_{\alpha \beta}=R_{(\alpha} S_{\beta)}$ with $R \cdot R=S \cdot S=0$ and $R \cdot S \gtrless 0$. It is easy to see that any vector in the (time-like) $R-S$ plane is an eigenvector with eigenvalue $\frac{1}{2} R \cdot S$ and that any vector in the orthogonal (space-like) plane is an eigenvector with eigenvalue zero.

\section{The Scalar Field}

A geometry whose source is a null electromagnetic field has a Ricci tensor belonging to class $A_{3 \ldots}$. If the electromagnetic field is non-null $R_{\alpha \beta}$ is in class $B_{1 b}$. If the source is a real scalar field [5] $R_{\alpha \beta}$ is in class $A_{-}$, if it is a complex scalar field [8] $R_{\alpha \beta}$ is in class $C_{-}$or $B_{1 b}$.

In this section we discuss the geometrization of a real massless scalar field in detail. The Lagrangean is given by

$$
L=\frac{1}{2} \phi^{, \alpha} \phi_{, \alpha}
$$

whence follow the equation of motion

$$
\phi_{; \alpha}^{, \alpha}=0
$$

and the stress-energy tensor

$$
T_{\alpha \beta}=\phi_{, \alpha} \phi_{. \beta}-\frac{1}{2} g_{\alpha \beta} \phi^{, \gamma} \phi_{. \gamma} .
$$

The Einstein equations then give us

where

$$
R_{\alpha \beta}=-\phi_{\alpha} \phi_{\beta}
$$

$$
\phi_{\alpha}=\phi_{, \alpha} .
$$

This Ricci tensor is in class $A_{-}$.

Conversely, if $R_{\alpha \beta}$ is in class $A_{\ldots}$ we can find a vector $\phi_{\alpha}$ such that $R_{\alpha \beta}$ differs from that given by Eq. (5.2) by a multiple of $g_{\alpha \beta}$. If in addition

$$
R=-\phi^{\alpha} \phi_{\alpha}
$$

then that multiple is zero and Eq. (5.2) is satisfied. The vector $\phi_{x}$ is determined by $R_{\alpha \beta}$ uniquely except for sign. If it obeys the equation

$$
\phi_{[\alpha ; \beta]}=0
$$

a scalar $\phi$ exists satisfying Eq. (5.3). Note that Eq. (5.5) is already in geometrical terms since $\phi_{\alpha}$ is determined by the geometry in an essentially unique way.

Writing Eqs. (5.3) and (5.5) in dyad component form we can easily show that by virtue of one of the contracted Bianchi identities the equation of motion, Eq. (5.1), gives no new condition on the geometry. 
In summary, a necessary and sufficient condition for a geometry to have as its source a massless real scalar field is that $R_{\alpha \beta}$ belong to class $A_{-}$, that $R$ be given in terms of the associated vector $\phi_{\alpha}$ by Eq. (5.4) and that $\phi_{\alpha}$ satisfy Eq. (5.5).

Let us compare these conditions with those given by Brill [4] and Penney [5]. In our notation the conditions found by Penney are

$$
\begin{gathered}
R_{\alpha}^{\beta} R_{\beta}^{\gamma}=R R_{\alpha}^{\gamma}, \\
\left(R_{\alpha \beta}-\frac{1}{2} R g_{\alpha \beta}\right) \omega^{\alpha} \omega^{\beta} \leqq 0 \quad \text { for any time-like vector } \omega^{\alpha}, \\
R^{\gamma \delta} R_{\gamma[\alpha ; \beta]}-\frac{1}{2} R_{[\alpha}^{\delta} R_{, \beta]}=0, \\
R>0 .
\end{gathered}
$$

Brill states Eqs. (5.6) and (5.8) only, but appears to assume Eq. (5.9) as well.

Writing Eq. (5.6) in dyad component form and choosing (without loss of generality) a frame in which $\Phi_{02}$ vanishes we find after a somewhat lengthy calculation that

$$
\Phi_{01}=\Phi_{12}=0, \quad R=-8 \Phi_{11}, \quad 4 \Phi_{11}^{2}=\Phi_{00} \Phi_{22} .
$$

From these equations it is easy to show that there exists a vector $\phi_{\alpha}$ determined uniquely up to sign and satisfying

$$
R_{\alpha \beta}= \pm \phi_{\alpha} \phi_{\beta} .
$$

Therefore, Eq. (5.6) is equivalent to the statement that $R_{\alpha \beta}$ belongs to class $A_{ \pm}$with the trace of the corresponding vector $\phi_{\alpha}$ satisfying

$$
R= \pm \phi_{\alpha} \phi^{\alpha} .
$$

Writing Eq. (5.7) in dyad component form we find that it states that $R_{\alpha \beta}$ belongs to $A_{-}$, assuming that Eq. (5.6) is valid.

Again assuming that Eq. (5.6) holds Eqs. (5.5) and (5.8) can be shown to be equivalent provided $R$ does not vanish. Eq. (5.8) is empty when $R$ is zero. However, as there is no reason why $R$ should not be zero (or negative) the condition given by Eq. (5.9) is not necessary and should be dropped. The statement in Ref. [9] referred to by Penney [8] applies only to systems of particles and their electromagnetic interactions.

Since we have seen that Eq. (5.5) is a condition on the geometry, necessary and sufficient conditions that a given geometry have as its source a massless real scalar field are given by Eqs. (5.6), (5.7) and (5.5). 
Acknowledgments. One of us (G. L.) would like to thank Professor W. Israel for very helpful discussions. We would also like to express our gratitude to Professor A. H. Taub for some valuable suggestions.

\section{Appendix}

The spinor equivalent $T_{A \dot{X}}$ of a real vector $T_{\alpha}$ is Hermitian and has dyad components $\left(T_{0}, T_{1}, T_{2}\right)$ defined by

$$
\begin{aligned}
& T_{0}=T_{A \dot{X}} k^{A} \bar{k}^{\dot{X}}=T_{\alpha} k^{\alpha}, \\
& T_{1}=T_{A \dot{X}} k^{A} \bar{m}^{\dot{X}}=T_{\alpha} t^{\alpha}, \\
& T_{2}=T_{A \dot{X}} m^{A} \bar{m}^{\dot{X}}=T_{\alpha} m^{\alpha},
\end{aligned}
$$

where $T_{0}$ and $T_{2}$ are real, $T_{1}$ is complex, and $\left\{k^{\alpha}, t^{\alpha}, \bar{t}^{\alpha}, m^{\alpha}\right\}$ is the null tetrad associated with the dyad $\left\{k^{A}, m^{A}\right\}$.

Choosing appropriate canonical forms for the dyad components of $R_{\alpha}$ and $S_{\alpha}$ we find with the aid of Eq. (3.3) the canonical forms for $R_{\alpha \beta}$. These are shown in Table 4 for classes $A, B$, and $C$. It should be noted that just as for the Petrov classification knowing the canonical form may not be sufficient to determine the class.

Table 4

\begin{tabular}{lll}
\hline Class & Canonical forms for the relevant vectors & $\begin{array}{l}\text { Non-zero components } \\
\text { of } S_{\alpha \beta}\end{array}$ \\
\hline$A_{1 \pm}$ & $\left(0, R_{1}, 0\right)$ & $\Phi_{11} \lessgtr 0, \Phi_{02} \neq 0$ \\
$A_{2 \pm}$ & $\left(R_{0}, 0, R_{2}\right), R_{0} R_{2}>0$ & $\Phi_{00}, \Phi_{11}, \Phi_{22} \lessgtr 0$ \\
$A_{3 \pm}$ & $\left(0,0, R_{2}\right)$ & $\Phi_{22} \lessgtr 0$ \\
$B_{1 a, b}$ & $\left(R_{0}, 0,0\right),\left(0,0, S_{2}\right), R_{0} S_{2} \gtrless 0$ & $\Phi_{11} \lessgtr 0$ \\
$B_{2 a}$ & $\left(0, R_{1}, 0\right),\left(0,0, S_{2}\right)$ & $\Phi_{12} \neq 0$ \\
$B_{2 b, c}$ & $\left(R_{0}, R_{1}, 0\right),\left(0,0, S_{2}\right), R_{0} S_{2} \gtrless 0$ & $\Phi_{11} \lessgtr 0, \Phi_{12} \neq 0$ \\
$B_{3 a, b, c}$ & $\left(0, R_{1}, 0\right),\left(0, S_{1}, 0\right), R_{1} \bar{S}_{1}+\bar{R}_{1} S_{1} \bar{\lessgtr}_{0}$ & $\Phi_{11} \sum 0, \Phi_{02} \neq 0$ \\
$B_{3 d, e}$ & $\left(0, R_{1}, R_{2}\right),\left(0, S_{1}, 0\right), R_{1} \bar{S}_{1}+\bar{R}_{1} S_{1} \lessgtr 0$ & $\Phi_{11} \gtrless 0, \Phi_{02}, \Phi_{12} \neq 0$ \\
$B_{3 f . g}$ & $\left(R_{0}, R_{1}, R_{2}\right),\left(0, S_{1}, 0\right), R_{0} R_{2}<R_{1} \bar{R}_{1}$, & $\Phi_{11} \gtrless 0, \Phi_{01}, \Phi_{02}, \Phi_{12} \neq 0$ \\
$B_{4 a, b}$ & $R_{1} \bar{S}_{1}+\bar{R}_{1} S_{1} \lessgtr 0$ & \\
$B_{5 a, b}$ & $\left(0,0, R_{2}\right),\left(S_{0}, 0, S_{2}\right), S_{0} S_{2}>0, R_{2} S_{0} \gtrless 0$ & $\Phi_{11}, \Phi_{22} \lessgtr 0$ \\
$B_{6 a}$ & $\left(R_{0}, 0, R_{2}\right),\left(S_{0}, 0, S_{2}\right), R_{0} R_{2}>0, S_{0} S_{2}>0$, & $\Phi_{00}, \Phi_{11}, \Phi_{22} \lessgtr 0$ \\
$B_{6 b, c}$ & $R_{0} S_{2}+R_{2} S_{0} \gtrless 0$ & \\
& $\left(0, R_{1}, 0\right),\left(S_{0}, 0, S_{2}\right), S_{0} S_{2}>0$ & $\Phi_{01} \neq 0, \Phi_{12} \neq 0$ \\
$C_{1 \pm}$ & $\left(0, R_{1}, 0\right),\left(S_{0}, S_{1}, S_{2}\right), S_{0} S_{2}>S_{1} \bar{S}_{1}$, & $\Phi_{11} \gtrless 0, \Phi_{01}, \Phi_{02}, \Phi_{12} \neq 0$ \\
$C_{2 \pm}$ & $R_{1} \bar{S}_{1}+\bar{R}_{1} S_{1} \lessgtr 0$ & \\
$C_{3 \pm}$ & $\left(0, S_{1}, 0\right),\left(T_{0}, 0, T_{2}\right), T_{0} T_{2}>0$ & $\Phi_{00}, \Phi_{11}, \Phi_{22} \lessgtr 0, \Phi_{02} \neq 0$ \\
\hline
\end{tabular}




\title{
References
}

1. Ludwig, G.: Commun. math. Phys. 17, 98 (1970).

2. Penrose, R.: Ann. Phys. 10, 171 (1960).

3. Schild, A.: Lectures on general relativity theory. (Proceedings of the Summer Seminar, Ithaca, New York, 1965, edited by J. Ehlers) Am. Math. Soc., Providence, R.I., 1967.

4. Brill, D.: Nuovo Cimento Suppl. 2, 1 (1964).

5. Penney, R.: Phys. Letters 11, 228 (1964).

6. Petrov, A. Z.: Perspectives in geometry and relativity. Edited by B. Hoffmann. Indiana: University Press 1966.

7. Pirani, F. A. E.: Lectures on general relativity. (Brandeis Summer Institute, 1964). Englewood Cliffs, N. J.: Prentice Hall 1965.

8. Penney, R.: J. Math. Phys. 7, 479 (1966).

9. Landau, L. D., Lifshitz, E.: The classical theory of fields. 2nd ed., p. 89. Addison-Wesley 1959

\author{
Garry Ludwig \\ Department of Mathematics \\ University of Alberta \\ Edmonton 7, Alberta, Canada
}

